\title{
SYMMETRY ANALYSIS AND SOLITARY WAVE SOLUTIONS OF NONLINEAR ION-ACOUSTIC WAVES EQUATION
}

\author{
M.H.M. MOUSSA ${ }^{1,3}$, A.A. GABER ${ }^{2,3 *}$ \\ ${ }^{1}$ Department of Mathematics, University Colledge, Umm Al-Qura University, Al-Leth, Suadi Arabia \\ ${ }^{2}$ Department of Mathematics, College of Science and Human Studies at Hotat Sudair, Majmaah \\ University, 11952, Suadi Arabia \\ ${ }^{3}$ Department of Mathematics, Faculty of Education, Ain Shams University, Roxy, Hiliopolis, Cairo, Egypt \\ *Corresponding author: aagaber6@gmail.com, a.gaber@mu.edu.sa

\begin{abstract}
The problem of nonlinear ion-acoustic waves equation in a magnetized plasma, known as
Zakharov-Kuznetsov equation, is investigated by using symmetry analysis. The carryover of the symmetry analysis has led to certain similarity reductions of this equation. Furthermore, exact solutions of similarity reductions are obtained by modified Exp-Function method with computational symbolic. Some figures are obtained to show the properties of the solutions.
\end{abstract}

\section{INTRODUCTION}

There are many well-known methods to obtain exact solutions [1-5]. In order to unite and widen various specialized solution method for partial differential equations Lie introduced the notion of continuous groups now know as Lie groups. contiuing his investigations he shown that partial differential equation can be reduced to many ordinary differential equations which is led to varied solutions. In the last century, the application of the Lie groups has been developed by a number of reserchers. Ovsiannikov [6], Olver [7], Ibragimov [8], and Bluman et al. [9] are some of the mathematicians who have huge number of studies in that field.

Received September $11^{\text {th }}, 2019$; accepted October $9^{\text {th }}, 2019$; published May $1^{\text {st }}, 2020$.

2010 Mathematics Subject Classification. 35B06.

Key words and phrases. symmetry analysis; modified Exp-Function method; Zakharov-Kuznetsov equation.

(C)2020 Authors retain the copyrights of their papers, and all open access articles are distributed under the terms of the Creative Commons Attribution License. 
Consider the nonlinear ion-acoustic waves equation which is called (1+3)-dimensional Zakharov-Kuznetsov (Zk) equation $[10,11]$ in the following form:

$$
u_{t}+p_{1} u u_{x}+p_{2} u_{x, x, x}+p_{3} u_{x, y, y}+p_{4} u_{x, z, z}=0
$$

where $\mathrm{p}_{1}, \mathrm{p}_{2}, \mathrm{p}_{3}$ and $\mathrm{p}_{4}$ are nonzero constants. ZK [10] is described the diffusion of nonlinear ion-acoustic waves in magnetized plasma [10]. This equation was devoted to study many properties including presence and stability of solitary wave solutions for the ZK model [10, $13-15]$.

\section{Determination OF THE SYMmetries}

Firstly, we shall conclude the similarity reductions using Lie group method [16 - 22]. In order to apply Lie group method we can write the one parameter Lie group of infinitesimal transformations as follow:

$$
\begin{aligned}
& t^{*}=t+\varepsilon A(t, x, y, z, u)+o\left(\varepsilon^{2}\right), x^{*}=x+\varepsilon B(t, x, y, z, u)+o\left(\varepsilon^{2}\right), \\
& y^{*}=y+\varepsilon C(t, x, y, z, u)+o\left(\varepsilon^{2}\right), z^{*}=z+\varepsilon D(t, x, y, z, u)+o\left(\varepsilon^{2}\right), \\
& u^{*}=u+\varepsilon E(t, x, y, z, u)+o\left(\varepsilon^{2}\right) .
\end{aligned}
$$

If we set

$$
\Delta=u_{t}+p_{1} u u_{x}+p_{2} u_{x, x, x}+p_{3} u_{x, y, y}+p_{4} u_{x, z, z}=0
$$

where subscripts $t, x, y$ and $z$ to the function $u$ denote differentiation with respect to these variables. The infinitesimal generator $\mathrm{V}$ associated with the above mentioned group of transformations can be presented as following expression

$$
V=A \frac{\partial}{\partial t}+B \frac{\partial}{\partial x}+C \frac{\partial}{\partial y}+D \frac{\partial}{\partial z}+E \frac{\partial}{\partial u}
$$

when the following invariance condition is satisfied:

$$
\Gamma^{(3)}(\Delta)=0
$$

where $\Gamma^{(3)}$ is the third order prolongation of the operator $\mathrm{V}$

$$
\Gamma^{(3)}=V+E_{[x]} \frac{\partial}{\partial u_{x}}+E_{[t]} \frac{\partial}{\partial u_{t}}+E_{[x x x]} \frac{\partial}{\partial u_{x x x}}+E_{[x y y]} \frac{\partial}{\partial u_{x y y}}+E_{[x z z]} \frac{\partial}{\partial u_{x z z}}
$$

where the components $E_{[x]}, E_{[x x]}, E_{[x y y]}, E_{[x z]}, E_{[t]} \ldots$. can be determined from the following expressions:

$$
\begin{gathered}
E_{[x]}=D_{x} E-u_{t} D_{x} A-u_{x} D_{x} B \\
E_{[x t]}=D_{t} E_{[s]}-u_{t x} D_{t} A-u_{x x} D_{t} B .
\end{gathered}
$$


Substituting (3) into invariance condition (5), yields an identity components $A_{x}, A_{x x}, B_{t}, B_{x}, \ldots$. hence we collect the coefficients of $u_{x}, u_{x, x, x}, \ldots$ and equate it to zero, which led to obtain a system of linear differential equations of the infinitesimals $A, B, C$ and $E$

$$
\begin{gathered}
A_{x}=A_{y}=A_{z}=A_{u}=A_{t, t}=0, \\
B_{y}=B_{z}=B_{u}=B_{t, t}=0, B_{x}=\frac{1}{3} A_{t}, \\
C_{t}=C_{x}=B_{u}=0, C_{y}=\frac{1}{3} A_{t}, C_{z}=\frac{-p_{3}}{p_{4}} D_{y}, \\
D_{t}=D_{x}=D_{u}=D_{y, y}=0, D_{z}=\frac{1}{3} A_{t}, \\
-p_{1}^{2} u^{2} B_{x}+p_{1} u A_{x}-p_{1} u B_{x}-p_{1} E+A_{t}=0, \\
3 p_{2} C_{x, x}+p_{3} C_{y, y}+p_{4} C_{z, z}=0,
\end{gathered}
$$

Solving resulting of partial differential equations system, we got:

$$
\begin{array}{rlrl}
A & =c_{1} t+c_{2}, & B & =\frac{1}{3} c_{1} x+p_{1} c_{7} t+c_{4} \\
C & =\frac{1}{3} c_{1} y-c_{3} \frac{p_{3}}{p_{4}} z+c_{5}, & D=\frac{1}{3} c_{1} z+c_{3} y+c_{6}, \\
E & =\frac{-2}{3} c_{1} u+c_{7} . &
\end{array}
$$

We can be easily write the vector field operator V from (9) as

$$
V=V_{1}\left(c_{1}\right)+V_{2}\left(c_{2}\right)+V_{3}\left(c_{3}\right)+V_{4}\left(c_{4}\right)+V_{5}\left(c_{5}\right)+V_{6}\left(c_{6}\right)+V_{7}\left(c_{7}\right),
$$

where

$$
\begin{aligned}
& V_{1}=t \frac{\partial}{\partial t}+\frac{1}{3}\left(x \frac{\partial}{\partial x}+y \frac{\partial}{\partial y}+z \frac{\partial}{\partial z}-2 u \frac{\partial}{\partial u}\right), \\
& V_{2}=\frac{\partial}{\partial t}, \quad V_{3}=\frac{-p_{3}}{p_{4}} z \frac{\partial}{\partial y}+y \frac{\partial}{\partial z} \\
& V_{4}=\frac{\partial}{\partial x}, \quad V_{5}=\frac{\partial}{\partial y}, \quad V_{6}=\frac{\partial}{\partial z}, \\
& V_{7}=p_{1} t \frac{\partial}{\partial x}+\frac{\partial}{\partial u} .
\end{aligned}
$$

The commutator relations are given in Table 1. 
Table 1: The commutator table

\begin{tabular}{cccccccc}
\hline & $V_{1}$ & $V_{2}$ & $V_{3}$ & $V_{4}$ & $V_{5}$ & $V_{6}$ & $V_{7}$ \\
\hline$V_{1}$ & 0 & $-V_{2}$ & 0 & $\frac{-1}{3} V_{4}$ & $\frac{-1}{3} V_{5}$ & $\frac{-1}{3} V_{6}$ & $\frac{2}{3} V_{7}$ \\
$V_{2}$ & $V_{2}$ & 0 & 0 & 0 & 0 & 0 & $p_{1} V_{4}$ \\
$V_{3}$ & 0 & 0 & 0 & 0 & $-V_{6}$ & $\frac{p_{3}}{p_{4}} V_{5}$ & 0 \\
$V_{4}$ & $\frac{1}{3} V_{4}$ & 0 & 0 & 0 & 0 & 0 & 0 \\
$V_{5}$ & $\frac{1}{3} V_{5}$ & 0 & 0 & $V_{6}$ & 0 & 0 & 0 \\
$V_{6}$ & $\frac{1}{3} V_{6}$ & 0 & $\frac{-p_{3}}{p_{4}} V_{5}$ & 0 & 0 & 0 & 0 \\
$V_{7}$ & $\frac{-2}{3} V_{7}$ & $-p_{1} V_{4}$ & 0 & 0 & 0 & 0 & 0 \\
\hline
\end{tabular}

From the commutator relations in table 1, we utilized the following six non-equivalent possibilities of Lie algebra

$$
\begin{aligned}
& \text { (I) } V_{1}+m_{1} V_{2}+m_{2} V_{4}+m_{3} V_{5}+m_{4} V_{6}+m_{5} V_{7}, \\
& \text { (II) } V_{2}+m_{1} V_{4}+m_{2} V_{5}+m_{3} V_{6} \\
& \text { (III) } V_{4}+m_{1} V_{5}+m_{2} V_{7} \\
& \text { (IV) } V_{2}+m_{1} V_{5}+m_{2} V_{7} \\
& \text { (V) } V_{2}+m_{1} V_{4}+m_{2} V_{7} \\
& \text { (VI) } V_{2}+m_{1} V_{4}+m_{2} V_{5}
\end{aligned}
$$

\section{REDUCTIONS AND EXACT SOLUTIONS}

In order to obtain the invariant transformation, we can write the characteristic equation as follow

$$
\frac{d t}{A(t, x, y, z, u)}=\frac{d x}{B(t, x, y, z, u)}=\frac{d y}{C(t, x, y, z, u)}=\frac{d z}{D(t, x, y, z, u)}=\frac{d u}{E(t, x, y, z, u)} .
$$

This equation is solved for the above six cases the invariant variables, then the corresponding reductions to partial differential equations are obtained and by using the similarity transformations the govern partial differential equations reduced to ordinary differential equations 
Table 2: The invariant variables and their corresponding partial differential equations

\begin{tabular}{|c|c|c|c|c|c|}
\hline \multirow[t]{2}{*}{ Case } & \multicolumn{4}{|c|}{ The invariant variables } & \multirow[t]{2}{*}{ Corresponding partial differential equations } \\
\hline & $\zeta_{1}$ & $\zeta_{2}$ & $\zeta_{3}$ & $u$ & \\
\hline \multirow[t]{2}{*}{$\mathrm{I}(\mathrm{i})$} & $\frac{x-\frac{m_{5}}{2} p_{1} t+n}{\left(\mathrm{t}+\mathrm{m}_{1}\right)^{\frac{1}{3}}}$ & $\frac{y+n_{3}}{\left(\mathrm{t}+\mathrm{m}_{1}\right)^{\frac{1}{3}}}$ & $\frac{z+n_{4}}{\left(\mathrm{t}+\mathrm{m}_{1}\right)^{\frac{1}{3}}}$ & $\frac{F}{\left(\mathrm{t}+\mathrm{m}_{1}\right)^{\frac{2}{3}}}+\frac{m_{5}}{2}$ & \\
\hline & & & & & $-3 \mathrm{p}_{2} \mathrm{~F}_{\zeta_{1} \zeta_{1} \zeta_{1}}-3 \mathrm{p}_{3} \mathrm{~F}_{\zeta_{1} \zeta_{2} \zeta_{2}}-3 \mathrm{p}_{4} \mathrm{~F}_{\zeta_{1} \zeta_{3} \zeta_{3}}=0$ \\
\hline \multirow[t]{2}{*}{ Case } & \multicolumn{4}{|c|}{ The invariant variables } & Corresponding partial differential equations \\
\hline & $\zeta_{1}$ & $\zeta_{2}$ & $\zeta_{3}$ & $u$ & \\
\hline I(ii) & $\frac{x-\frac{m_{5}}{2} p_{1} t+n}{\left(\mathrm{t}+\mathrm{m}_{1}\right)^{\frac{1}{3}}}$ & $\frac{y+n_{3}}{\left(\mathrm{t}+\mathrm{m}_{1}\right)^{\frac{1}{3}}}$ & $\frac{z+n_{4}}{\left(\mathrm{t}+\mathrm{m}_{1}\right)^{\frac{1}{3}}}$ & $\frac{F}{\left(\mathrm{t}+\mathrm{m}_{1}\right)^{\frac{2}{3}}}+\frac{m_{5}}{2}$ & $\begin{array}{l}\text { If we put } \mathrm{p}_{1} \mathrm{FF}_{\zeta_{1}}+\left(\mathrm{p}_{2} \mathrm{~F}_{\zeta_{1} \zeta_{1} \zeta_{1}}+\mathrm{p}_{3} \mathrm{~F}_{\zeta_{2} \zeta_{2}}\right. \\
\left.+\mathrm{p}_{4} \mathrm{~F}_{\zeta_{3} \zeta_{3}}\right)_{\zeta_{1}}=0 \text {, we conclude that } \\
{\left[2 \mathrm{~F}+\zeta_{1} \mathrm{~F}_{\zeta_{1}}+\zeta_{2} \mathrm{~F}_{\zeta_{2}}+\zeta_{3} \mathrm{~F}_{\zeta_{3}}\right]=0}\end{array}$ \\
\hline II & $x-m_{1} t$ & $y-m_{2} t$ & $\mathrm{z}-\mathrm{m}_{3} \mathrm{t}$ & $F$ & $\begin{array}{l}m_{1} \mathrm{~F}_{\zeta_{1}}+m_{2} \mathrm{~F}_{\zeta_{2}}+m_{3} \mathrm{~F}_{\zeta_{3}}-\mathrm{p}_{1} \mathrm{FF}_{\zeta_{1}} \\
-\mathrm{p}_{2} \mathrm{~F}_{\zeta_{1} \zeta_{1} \zeta_{1}}-\mathrm{p}_{3} \mathrm{~F}_{\zeta_{1} \zeta_{2} \zeta_{2}}-\mathrm{p}_{4} \mathrm{~F}_{\zeta_{1} \zeta_{3} \zeta_{3}}=0\end{array}$ \\
\hline III & $t$ & $y-m_{1} x$ & $\mathrm{z}-\mathrm{m}_{2} \mathrm{x}$ & $F$ & 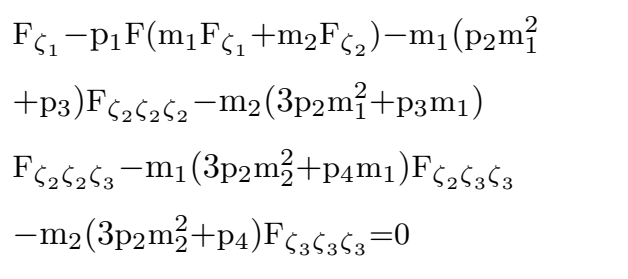 \\
\hline IV & $x$ & $\mathrm{y}-\mathrm{m}_{1} \mathrm{t}$ & $\mathrm{z}-\mathrm{m}_{2} \mathrm{t}$ & $F$ & $\begin{array}{l}m_{1} \mathrm{~F}_{\zeta_{2}}+m_{2} \mathrm{~F}_{\zeta_{3}}-\mathrm{p}_{1} \mathrm{FF}_{\zeta_{1}}-\mathrm{p}_{2} \mathrm{~F}_{\zeta_{1} \zeta_{1} \zeta_{1}} \\
-\mathrm{p}_{3} \mathrm{~F}_{\zeta_{1} \zeta_{2} \zeta_{2}}-\mathrm{p}_{4} \mathrm{~F}_{\zeta_{1} \zeta_{3} \zeta_{3}}=0\end{array}$ \\
\hline $\mathrm{V}$ & $\mathrm{x}-\mathrm{m}_{1} \mathrm{t}$ & $\mathrm{y}$ & $\mathrm{z}-\mathrm{m}_{2} \mathrm{t}$ & $F$ & $\begin{array}{l}m_{1} \mathrm{~F}_{\zeta_{1}}+m_{2} \mathrm{~F}_{\zeta_{3}}-\mathrm{p}_{1} \mathrm{FF}_{\zeta_{1}}-\mathrm{p}_{2} \mathrm{~F}_{\zeta_{1} \zeta_{1} \zeta_{1}} \\
-\mathrm{p}_{3} \mathrm{~F}_{\zeta_{1} \zeta_{2} \zeta_{2}}-\mathrm{p}_{4} \mathrm{~F}_{\zeta_{1} \zeta_{3} \zeta_{3}}=0\end{array}$ \\
\hline VI & $\mathrm{x}-\mathrm{m}_{1} \mathrm{t}$ & $y-m_{2} t$ & $z$ & $F$ & $\begin{array}{l}m_{1} \mathrm{~F}_{\zeta_{1}}+m_{2} \mathrm{~F}_{\zeta_{2}}-\mathrm{p}_{1} \mathrm{FF}_{\zeta_{1}}-\mathrm{p}_{2} \mathrm{~F}_{\zeta_{1} \zeta_{1} \zeta_{1}} \\
-\mathrm{p}_{3} \mathrm{~F}_{\zeta_{1} \zeta_{2} \zeta_{2}}-\mathrm{p}_{4} \mathrm{~F}_{\zeta_{1} \zeta_{3} \zeta_{3}}=0\end{array}$ \\
\hline
\end{tabular}

where $\mathrm{n}_{3}=3 m_{3}, n_{4}=3 m_{4}, n=3 m_{2}-\frac{9}{2} p_{1} m_{2} m_{5}$ 


\section{Case I(i):}

In this case, we put $\theta=k_{1} \zeta_{1}+k_{2} \zeta_{2}+k_{3} \zeta_{3}$, then, the equation can be written in the form:

$$
2 F+\theta F^{\prime}-3 p_{1} k_{1} F F^{\prime}-3 k_{1}\left(p_{2} k_{1}^{2}+p_{3} k_{2}^{2}+p_{4} k_{3}^{2}\right) F^{\prime \prime \prime}=0 .
$$

To obtain the solution for the ODE corresponding to this case, we assume that this solution takes the following form

$$
F=a_{0}+a_{1} \theta+a_{2} \theta^{2}+\frac{b_{1}}{\theta}+\frac{b_{2}}{\theta^{2}} .
$$

Substituting Eq. (14) into Eq. (13), equating to zero the coefficients of all powers of $\theta$ yields a set of algebraic equations for $a_{0}, a_{1}, a_{1}, b_{1}, b_{2}$,solving the system of algebraic equations with the aid of Maple, we obtain the following results:- $a_{0}=a_{1}=b_{1}=b_{2}=0, a_{1}=\frac{1}{\mathrm{k}_{1} \mathrm{p}_{1}}$, then, the final solution of Eq. (1) can be written in the form:

$$
u(t, x, y, z)=\frac{1}{\mathrm{k}_{1} \mathrm{p}_{1}\left(\mathrm{t}+\mathrm{m}_{1}\right)}\left[k_{1}\left(x-\frac{m_{5}}{2} p_{1} t+n\right)+k_{2}\left(y+n_{3}\right)+k_{3}\left(z+n_{4}\right)\right]+\frac{m_{5}}{2} .
$$

Case I(ii): In this case we have to solve the following two PDEs

$$
\begin{array}{r}
p_{1} F F_{\zeta_{1}}+p_{2} F_{\zeta_{1} \zeta_{1} \zeta_{1}}+p_{3} F_{\zeta_{1} \zeta_{2} \zeta_{2}}+p_{4} F_{\zeta_{1} \zeta_{3} \zeta_{3}}=0 \\
2 F+\zeta_{1} F_{\zeta_{1}}+\zeta_{2} F_{\zeta_{2}}+\zeta_{3} F_{\zeta_{3}}=0
\end{array}
$$

We now introduce the simplified form of Lie-group transformations namely, the scaling group of transformation

$$
F=e^{\epsilon} \bar{F}, \quad \zeta_{1}=e^{\epsilon_{1}} \bar{\zeta}_{1}, \quad \zeta_{2}=e^{\epsilon_{2}} \bar{\zeta}_{2}, \quad \zeta_{3}=e^{\epsilon_{3}} \bar{\zeta}_{3}
$$

Substituting from (18) into (17) we have $\epsilon_{1}=\epsilon_{2}=\epsilon_{3}=-\epsilon$.

This mean that (16) is invariant under the transformation (18) and the characteristic equation can be written as

$$
\frac{d \zeta_{1}}{\zeta_{1}}=\frac{d \zeta_{2}}{\zeta_{2}}=\frac{d \zeta_{3}}{\zeta_{3}}=\frac{-d F}{F}
$$

We get the similarity variables

$$
\eta_{1}=\frac{\zeta_{1}}{\zeta_{2}}, \quad \eta_{2}=\frac{\zeta_{3}}{\zeta_{2}}, \quad F=\frac{f}{\zeta_{2}^{2}}
$$

Substituting from (20) into (17) we find that it is satisfied. Also substituting from (20) to Eq.(16) we obtain

$$
\begin{aligned}
& p_{1} f f_{\eta_{1}}+p_{2} f_{\eta_{1} \eta_{1} \eta_{1}}+12 p_{3} f_{\eta_{1}}+8 p_{3} \eta_{1} f_{\eta_{1}}+8 p_{3} \eta_{2} f_{\eta_{2}} \\
& p_{3} \eta_{1}^{2} f_{\eta_{1} \eta_{1} \eta_{1}}+p_{3} \eta_{2}^{2} f_{\eta_{1} \eta_{1} \eta_{2}}+2 p_{3} \eta_{1} \eta_{2} f_{\eta_{1} \eta_{2} \eta_{2}}+p_{4} f_{\eta_{1} \eta_{2} \eta_{2}}=0 .
\end{aligned}
$$

By using $\theta=\eta_{1}+\eta_{2}(21)$ can be written in form

$$
p_{1} f f^{\prime}+p_{2} f^{\prime \prime \prime}+4 p_{3}(3+2 \theta) f^{\prime}+\left(p_{3} \theta^{2}+p_{4}\right) f^{\prime \prime \prime}+4 \theta+\theta^{2}+2-\theta=0 .
$$


Using the same method in the previous case, hence, we have obtained the following exact solution to ODE corresponding this case in the form $f=\frac{-12\left(p_{2}+p_{4}\right)}{p_{1} \theta^{2}}$, then the solution of Zk equation is

$$
u(t, x, z)=\frac{m_{5}}{2}-\frac{12\left(p_{2}+p_{4}\right)}{p_{1}\left(n+n_{4}-\frac{m_{5}}{2} t+x+z\right)^{2}} .
$$

Case II: We take $\theta=k_{1} \zeta_{1}+k_{2} \zeta_{2}+k_{3} \zeta_{3}$, then equation of case (II) can be written in the form

$$
\begin{aligned}
& \left(k_{1} m_{1}+k_{2} m_{2}+k_{3} m_{3}\right) F^{\prime}-p_{1} k_{1} F F^{\prime} \\
& -k_{1}\left(p_{2} k_{1}^{2}+p_{3} k_{2}^{2}+p_{4} k_{3}^{2}\right) F^{\prime \prime \prime}=0 .
\end{aligned}
$$

To utilize the solution for the ODE corresponding to this case, we used modified Exp-Function method [13,23], which is expressed in the form:

$$
\begin{aligned}
F(\theta) & =\frac{\sum_{n=-c}^{p} a_{n}[\phi(\theta)]^{n}}{\sum_{m=-d}^{q} b_{m}[\phi(\theta)]^{m}} \\
& =\frac{a_{-c}[\phi(\theta)]^{-c}+\ldots+a_{p}[\phi(\theta)]^{p}}{b_{-d}[\phi(\theta)]^{-d}+\ldots+b_{q}[\phi(\theta)]^{q}}
\end{aligned}
$$

where $\phi(\theta)$ satisfies the following Riccati equation

$$
\phi^{\prime}(\theta)=A+B \phi(\theta)+C \phi^{2}(\theta)
$$

see $[24-25]$.

We can freely choose the values of $n$ and $m$ in (25), that the solution does not depend on the balancing of the highest order linear and nonlinear terms [24].

$$
\begin{gathered}
F^{\prime \prime \prime}=\frac{a_{1}[\phi(\theta)]^{-c-8 d-3}+\ldots+a_{2}[\phi(\theta)]^{p+8 d+3}}{b_{1}[\phi(\theta)]^{-9 d}+\ldots+b_{2}[\phi(\theta)]^{9 q}}, \\
F F^{\prime}=\frac{a_{3}[\phi(\theta)]^{-2 c-7 d-3}+\ldots+a_{4}[\phi(\theta)]^{2 p+7 d+3}}{b_{3}[\phi(\theta)]^{-9 d}+\ldots+b_{4}[\phi(\theta)]^{9 q}},
\end{gathered}
$$

where $\mathrm{a}_{i}$ and $\mathrm{b}_{i}$ are determined coefficients only for simplicity. From balancing the lowest order and highest order of $\phi(27-28)$ we obtain $-c-8 d-3=-2 c-7 d-3$, which leads to the limit $c=d$ and $p+8 d+3=2 p+7 d+3$, which leads to the limit $p=q$. For simplicity, we set $\mathrm{p}=\mathrm{q}=1$, we have

$$
\begin{aligned}
F & =\frac{a_{-1}[\phi(\theta)]^{-1}+a_{0}+a_{1}[\phi(\theta)]}{b_{-1}[\phi(\theta)]^{-1}+b_{0}+b_{1}[\phi(\theta)]} \\
& =\frac{a_{-1}+a_{0}[\phi(\theta)]+a_{1}[\phi(\theta)]^{2}}{b_{-1}+b_{0}[\phi(\theta)]+b_{1}[\phi(\theta)]^{2}}
\end{aligned}
$$


Substituting (29) into (24), equating to zero the coefficients of all powers of $\phi(\theta)$ yields a set of algebraic equations for $\mathrm{a}_{i}$ and $\mathrm{b}_{i}$. By aid Maple we solve this algebraic equations, we get:

$$
\begin{aligned}
a_{-1} & =\left[\frac { 1 } { k _ { 1 } p _ { 1 } } \left(k_{1} m_{1}+k_{2} m_{2}+k_{3} m_{3}\right.\right. \\
& \left.\left.-k_{1}\left(B^{2}+8 A C\right)\left(p_{2} k_{1}^{2}+p_{3} k_{2}^{2}+p_{4} k_{3}^{2}\right)\right)\right] b_{-1}, \\
a_{0} & =\left[\frac{-12 B C}{p_{1}}\left(p_{2} k_{1}^{2}+p_{3} k_{2}^{2}+p_{4} k_{3}^{2}\right)\right] b_{-1}, \\
a_{1} & =\left[\frac{-12 C^{2}}{p_{1}}\left(p_{2} k_{1}^{2}+p_{3} k_{2}^{2}+p_{4} k_{3}^{2}\right)\right] b_{-1}, \quad b_{0}=b_{1}=0 .
\end{aligned}
$$

The corresponding traveling wave solutions to (1) are:

Case $1: \mathrm{A} \neq 0, \mathrm{~B} \neq 0, \mathrm{C} \neq 0$.

$$
\begin{aligned}
u(t, x, y, z) & =\frac{1}{k_{1} p_{1}}\left(k_{1} m_{1}+k_{2} m_{2}+k_{3} m_{3}-k_{1}\left(B^{2}+8 A C\right)\left(p_{2} k_{1}^{2}+p_{3} k_{2}^{2}+p_{4} k_{3}^{2}\right)\right) \\
& -\frac{12 B C}{p_{1}}\left(p_{2} k_{1}^{2}+p_{3} k_{2}^{2}+p_{4} k_{3}^{2}\right)\left[\frac{-B}{2 C}+\frac{\sqrt{4 A C-B^{2}}}{2 C}\right. \\
& \left.\tan \left(\frac{1}{2}\left(\sqrt{4 A C-B^{2}}\left(k_{1}\left(\mathrm{x}-\mathrm{m}_{1} \mathrm{t}\right)+k_{2}\left(\mathrm{y}-\mathrm{m}_{2} \mathrm{t}\right)+k_{3}\left(\mathrm{z}-\mathrm{m}_{2} \mathrm{t}\right)+\mathrm{d}_{0}\right)\right)\right)\right] \\
& -\frac{12 C^{2}}{p_{1}}\left(p_{2} k_{1}^{2}+p_{3} k_{2}^{2}+p_{4} k_{3}^{2}\right)\left[\frac{-B}{2 C}+\frac{\sqrt{4 A C-B^{2}}}{2 C}\right. \\
& \left.\tan \left(\frac{1}{2}\left(\sqrt{4 A C-B^{2}}\left(k_{1}\left(\mathrm{x}-\mathrm{m}_{1} \mathrm{t}\right)+k_{2}\left(\mathrm{y}-\mathrm{m}_{2} \mathrm{t}\right)+k_{3}\left(\mathrm{z}-\mathrm{m}_{2} \mathrm{t}\right)+\mathrm{d}_{0}\right)\right)\right)\right]^{2} .
\end{aligned}
$$

Case 2: $\mathrm{A}=0, \mathrm{~B} \neq 0, \mathrm{C} \neq 0$.

$$
\begin{aligned}
u(t, x, y, z) & =\frac{1}{k_{1} p_{1}}\left(k_{1} m_{1}+k_{2} m_{2}+k_{3} m_{3}-k_{1}\left(B^{2}+8 A C\right)\left(p_{2} k_{1}^{2}+p_{3} k_{2}^{2}+p_{4} k_{3}^{2}\right)\right) \\
& -\frac{12 B C}{p_{1}}\left(p_{2} k_{1}^{2}+p_{3} k_{2}^{2}+p_{4} k_{3}^{2}\right)\left[\frac{-B \exp \left(B\left(k_{1}\left(\mathrm{x}-\mathrm{m}_{1} \mathrm{t}\right)+k_{2}\left(\mathrm{y}-\mathrm{m}_{2} \mathrm{t}\right)+k_{3}\left(\mathrm{z}-\mathrm{m}_{2} \mathrm{t}\right)\right)+B d_{0}\right)}{C \exp \left(B\left(k_{1}\left(\mathrm{x}-\mathrm{m}_{1} \mathrm{t}\right)+k_{2}\left(\mathrm{y}-\mathrm{m}_{2} \mathrm{t}\right)+k_{3}\left(\mathrm{z}-\mathrm{m}_{2} \mathrm{t}\right)\right)+B d_{0}\right)-1}\right] \\
& -\frac{12 C^{2}}{p_{1}}\left(p_{2} k_{1}^{2}+p_{3} k_{2}^{2}+p_{4} k_{3}^{2}\right)\left[\frac{-B \exp \left(B\left(k_{1}\left(\mathrm{x}-\mathrm{m}_{1} \mathrm{t}\right)+k_{2}\left(\mathrm{y}-\mathrm{m}_{2} \mathrm{t}\right)+k_{3}\left(\mathrm{z}-\mathrm{m}_{2} \mathrm{t}\right)\right)+B d_{0}\right)}{C \exp \left(B\left(k_{1}\left(\mathrm{x}-\mathrm{m}_{1} \mathrm{t}\right)+k_{2}\left(\mathrm{y}-\mathrm{m}_{2} \mathrm{t}\right)+k_{3}\left(\mathrm{z}-\mathrm{m}_{2} \mathrm{t}\right)\right)+B d_{0}\right)-1}\right]^{2} .
\end{aligned}
$$

Case 3: $\mathrm{A}=\frac{1}{2}, \mathrm{~B}=0, \mathrm{C}=\frac{1}{2}$.

$$
\begin{aligned}
u(t, x, y, z) & =\frac{1}{k_{1} p_{1}}\left(k_{1} m_{1}+k_{2} m_{2}+k_{3} m_{3}-2 k_{1}\left(p_{2} k_{1}^{2}+p_{3} k_{2}^{2}+p_{4} k_{3}^{2}\right)\right) \\
& -\frac{3}{p_{1}}\left(p_{2} k_{1}^{2}+p_{3} k_{2}^{2}+p_{4} k_{3}^{2}\right)\left[\frac{\tan \left(k_{1}\left(\mathrm{x}-\mathrm{m}_{1} \mathrm{t}\right)+k_{2}\left(\mathrm{y}-\mathrm{m}_{2} \mathrm{t}\right)+k_{3}\left(\mathrm{z}-\mathrm{m}_{2} \mathrm{t}\right)\right)}{1 \pm \sec \left(k_{1}\left(\mathrm{x}-\mathrm{m}_{1} \mathrm{t}\right)+k_{2}\left(\mathrm{y}-\mathrm{m}_{2} \mathrm{t}\right)+k_{3}\left(\mathrm{z}-\mathrm{m}_{2} \mathrm{t}\right)\right)}\right]^{2} .
\end{aligned}
$$


Case III: We have two subcases

Subcase(a) We take $\theta=k_{1} \zeta_{1}+k_{2} \zeta_{2}+k_{3} \zeta_{3}$, then equation of case (III) can be written in the form

$$
\begin{aligned}
& k_{1} F^{\prime}-p_{1}\left(k_{2} m_{1}+k_{3} m_{2}\right) F F^{\prime}-\left(k_{2} m_{1}+k_{3} m_{2}\right) \\
& \left(p_{2}\left(k_{2} m_{1}+k_{3} m_{2}\right)^{2}+p_{3} k_{2}^{2}+p_{4} k_{3}^{2}\right) F^{\prime \prime \prime}=0 .
\end{aligned}
$$

Substituting (29) into (34), equating to zero the coefficients of all powers of $\phi(\theta)$ yields a set of algebraic equations for $\mathrm{a}_{i}$ and $\mathrm{b}_{i}$ i.e $\mathrm{i}=-1: 1$. By aid Maple we solve this algebraic equations, yields

$$
\begin{aligned}
a_{1} & =b_{-1}=b_{0}=0, B=0, a_{0} \text { is arbitrary } \\
k_{1} & =\left(k_{2} m_{1}+k_{3} m_{2}\right)\left(a_{0} p_{1}+8 A C\left(k_{2} m_{1} k_{3} m_{2} p_{2}\right.\right. \\
& \left.\left.+k_{1}^{2}\left(m_{1}^{2} p_{2}+p_{3}\right)+k_{3}^{2}\left(m_{2}^{2} p_{3}+p_{4}\right)\right)\right), \\
a_{-1} & =\left[\frac { - 1 2 A ^ { 2 } } { p _ { 1 } } \left(k_{2} m_{1} k_{3} m_{2} p_{2}+k_{1}^{2}\left(m_{1}^{2} p_{2}+p_{3}\right)\right.\right. \\
& \left.\left.+k_{3}^{2}\left(m_{2}^{2} p_{3}+p_{4}\right)\right)\right] b_{1} .
\end{aligned}
$$

We apply the related $\phi(\theta)$ functions for this choice of A, B and C.

Using the cases in Appendix A wherein $\mathrm{A}=1, \mathrm{C}=1$, yields

$$
\begin{aligned}
u(t, x, y, z) & =a_{0}-\frac{12}{p_{1}}\left(k_{2} m_{1} k_{3} m_{2} p_{2}\right. \\
& \left.+k_{1}^{2}\left(m_{1}^{2} p_{2}+p_{3}\right)+k_{3}^{2}\left(m_{2}^{2} p_{3}+p_{4}\right)\right) \\
& \frac{1}{\tan ^{2}\left(k_{1} t+k_{2}\left(\mathrm{y}-\mathrm{m}_{1} \mathrm{x}\right)+k_{3}\left(\mathrm{z}-\mathrm{m}_{2} \mathrm{x}\right)\right)} .
\end{aligned}
$$

where $\mathrm{k}_{1}=\left(k_{2} m_{1}+k_{3} m_{2}\right)\left(a_{0} p_{1}+8\left(k_{2} m_{1} k_{3} m_{2} p_{2}+k_{1}^{2}\left(m_{1}^{2} p_{2}+p_{3}\right)+k_{3}^{2}\left(m_{2}^{2} p_{3}+p_{4}\right)\right)\right)$.

For $\mathrm{A}=\frac{1}{2}, \mathrm{C}=\frac{-1}{2}$, we get the following solutions

$$
\begin{aligned}
u(t, x, y, z) & =a_{0}+\frac{3}{p_{1}}\left(k_{2} m_{1} k_{3} m_{2} p_{2}\right. \\
& \left.+k_{1}^{2}\left(m_{1}^{2} p_{2}+p_{3}\right)+k_{3}^{2}\left(m_{2}^{2} p_{3}+p_{4}\right)\right) \\
& {\left[\frac{1 \pm \sec \mathrm{h}\left(k_{1} t+k_{2}\left(\mathrm{y}-\mathrm{m}_{1} \mathrm{x}\right)+k_{3}\left(\mathrm{z}-\mathrm{m}_{2} \mathrm{x}\right)\right)}{\tanh \left(k_{1} t+k_{2}\left(\mathrm{y}-\mathrm{m}_{1} \mathrm{x}\right)+k_{3}\left(\mathrm{z}-\mathrm{m}_{2} \mathrm{x}\right)\right)}\right]^{2} }
\end{aligned}
$$

where $\mathrm{k}_{1}=\left(k_{2} m_{1}+k_{3} m_{2}\right)\left(a_{0} p_{1}-2\left(k_{2} m_{1} k_{3} m_{2} p_{2}+k_{1}^{2}\left(m_{1}^{2} p_{2}+p_{3}\right)+k_{3}^{2}\left(m_{2}^{2} p_{3}+p_{4}\right)\right)\right)$

Subcase(b) Using the scaling transformation to case III

$$
F=e^{\epsilon} \bar{F}, \quad \zeta_{1}=e^{\epsilon_{1}} \bar{\zeta}_{1}, \quad \zeta_{2}=e^{\epsilon_{2}} \bar{\zeta}_{2}, \quad \zeta_{3}=e^{\epsilon_{3}} \bar{\zeta}_{3}
$$

Substituting from Eq.(38) into case III we have $\frac{-2}{3} \epsilon_{1}=-2 \epsilon_{2}=-2 \epsilon_{3}=\epsilon$.

Then, the characteristic equation can be written as

$$
\frac{-2}{3} \frac{d \zeta_{1}}{\zeta_{1}}=\frac{-2 d \zeta_{2}}{\zeta_{2}}=\frac{-2 d \zeta_{3}}{\zeta_{3}}=\frac{d F}{F}
$$


We get the similarity variables

$$
\eta_{1}=\frac{\zeta_{2}}{\zeta_{1}^{\frac{1}{3}}}, \quad \eta_{2}=\frac{\zeta_{3}}{\zeta_{1}^{\frac{1}{3}}}, \quad F=\frac{f}{\zeta_{1}^{\frac{2}{2}}}
$$

By substituting into case III we get

$$
\begin{aligned}
& \frac{2}{3} f+\frac{1}{3} \eta_{1} f_{\eta_{1}}+\frac{1}{3} \eta_{2} f_{\eta_{2}}+m_{1} p_{1} f f_{\eta_{1}}+m_{2} p_{1} f f_{\eta_{2}} \\
& +m_{1}\left(m_{1}^{2} p_{2}+p_{3}\right) f_{\eta_{1} \eta_{1} \eta_{1}}+m_{2}\left(3 m_{1}^{2} p_{2}+p_{3}\right) f_{\eta_{1} \eta_{1} \eta_{2}} \\
& +m_{1}\left(3 m_{2}^{2} p_{2}+p_{4}\right) f_{\eta_{1} \eta_{2} \eta_{2}}+m_{2}\left(m_{2}^{2} p_{2}+p_{4}\right) f_{\eta_{2} \eta_{2} \eta_{2}}=0
\end{aligned}
$$

By using $\theta=k_{1} \eta_{1}+k_{2} \eta_{2},(41)$ can be written in form

$$
\begin{aligned}
& \frac{2}{3} f+\frac{1}{3} \theta f^{\prime}+p_{1}\left(m_{1} k_{1}+m_{2} k_{2}\right) f f^{\prime} \\
& +m_{1} k_{1}^{3}\left(m_{1}^{2} p_{2}+p_{3}\right) f^{\prime \prime \prime}+m_{2} k_{1}^{2} k_{2}\left(3 m_{1}^{2} p_{2}+p_{3}\right) f^{\prime \prime \prime} \\
& +m_{1} k_{2}^{2} k_{1}\left(3 m_{2}^{2} p_{2}+p_{4}\right) f^{\prime \prime \prime}+m_{2} k_{2}^{3}\left(m_{2}^{2} p_{2}+p_{4}\right) f^{\prime \prime \prime}=0 .
\end{aligned}
$$

To find the solution for the ODE corresponding to this case, we assume that this solution takes the following form

$$
f=a_{0}+a_{1} \theta+a_{2} \theta^{2}+\frac{b_{1}}{\theta}+\frac{b_{2}}{\theta^{2}}
$$

where $a_{0}, a_{1}, a_{2}, b_{1}$ and $b_{2}$ are arbitrary constants, Substituting from (43) into (42) and collecting the various powers of $\theta$ then equating them to zero, we get system of algebraic equations in the constants $a_{0}, a_{1}, a_{2}$, $b_{1}$ and $b_{2}$. Solving this system with the aid of Maple program, we get the following solutions:

$$
\begin{aligned}
& a_{0}=a_{2}=b_{1}=b_{2}=0, \\
& k_{1}=\frac{-\left(1+a_{1} p_{1} m_{2} k_{2}\right)}{a_{1} p_{1} m_{1}} .
\end{aligned}
$$

Then, we have obtained the following new exact solution for (1)

$$
u(t, x, y, z)=\frac{a_{2}}{t}\left(k_{1}\left(\mathrm{y}-\mathrm{m}_{1} \mathrm{x}\right)+k_{2}\left(\mathrm{z}-\mathrm{m}_{2} \mathrm{x}\right)\right) .
$$

Cases IV: We take the transformation $\theta=k_{1} \zeta_{1}+k_{2} \zeta_{2}+k_{3} \zeta_{3}$, we get

$$
\begin{aligned}
& \left(k_{2} m_{1}+k_{3} m_{2}\right) F^{\prime}-p_{1} k_{1} F F^{\prime} \\
& -3 k_{1}\left(p_{2} k_{1}^{2}+p_{3} k_{2}^{2}+p_{4} k_{3}^{2}\right) F^{\prime \prime \prime}=0 .
\end{aligned}
$$

To obtain the solution for the ODE corresponding to this case, substituting (29) into (46), equating to zero the coefficients of all powers of $\phi(\theta)$ yields a set of algebraic equations for $\mathrm{a}_{0}, \mathrm{a}_{i}, \mathrm{~b}_{i}, \mathrm{i}=1,2$. By aid 
Maple we solve this algebraic equations, yields

$$
\begin{aligned}
a_{0} & =\frac{1}{12 p_{1} k_{1} A^{2}}\left(\mathrm{a}_{-1} k_{1} p_{1}\left(B^{2}+8 A C\right)+12 \mathrm{~b}_{1} A^{2}\left(m_{1} k_{2}+m_{2} k_{3}\right)\right) \\
p_{2} & =-\frac{1}{12 k_{1} A^{2}}\left(\frac{p_{1} \mathrm{a}_{-1}}{\mathrm{~b}_{1}}+12 A^{2}\left(p_{3} k_{2}^{2}+p_{4} k_{3}^{2}\right)\right), \mathrm{a}_{0}=\frac{\mathrm{a}_{-1} B}{p_{1}} \\
\mathrm{~b}_{-1} & =\mathrm{b}_{0}=0 .
\end{aligned}
$$

Using choices for A, B and C, then we obtain the follwing exact solutions of (1)

$$
\begin{aligned}
u(t, x, y, z) & =\frac{1}{3 p_{1} k_{1}}\left(2 \mathrm{a}_{-1} k_{1} p_{1}+3 \mathrm{~b}_{1}\left(m_{1} k_{2}+m_{2} k_{3}\right)\right) \\
& +\frac{\mathrm{a}_{-1}}{\mathrm{~b}_{1}\left(\tan \left(k_{1} x+k_{2}\left(\mathrm{y}-\mathrm{m}_{1} \mathrm{t}\right)+k_{3}\left(\mathrm{z}-\mathrm{m}_{2} \mathrm{t}\right)\right) \pm \sec \left(k_{1} x+k_{2}\left(\mathrm{y}-\mathrm{m}_{1} \mathrm{t}\right)+k_{3}\left(\mathrm{z}-\mathrm{m}_{2} \mathrm{t}\right)\right)\right)^{2}},
\end{aligned}
$$

where $\mathrm{A}=\frac{1}{2}, \mathrm{~B}=0, \mathrm{C}=\frac{1}{2}$ and $p_{2}=-\frac{1}{3 k_{1}}\left(\frac{p_{1} \mathrm{a}-1}{\mathrm{~b}_{1}}+3\left(p_{3} k_{2}^{2}+p_{4} k_{3}^{2}\right)\right)$.

$$
\begin{aligned}
u(t, x, y, z) & =\frac{1}{3 p_{1} k_{1}}\left(-2 \mathrm{a}_{-1} k_{1} p_{1}+3 \mathrm{~b}_{1}\left(m_{1} k_{2}+m_{2} k_{3}\right)\right) \\
& +\frac{\mathrm{a}_{-1}}{\mathrm{~b}_{1}\left(\tanh \left(k_{1} x+k_{2}\left(\mathrm{y}-\mathrm{m}_{1} \mathrm{t}\right)+k_{3}\left(\mathrm{z}-\mathrm{m}_{2} \mathrm{t}\right)\right) \pm \operatorname{sech}\left(k_{1} x+k_{2}\left(\mathrm{y}-\mathrm{m}_{1} \mathrm{t}\right)+k_{3}\left(\mathrm{z}-\mathrm{m}_{2} \mathrm{t}\right)\right)\right)^{2}},
\end{aligned}
$$

where $\mathrm{A}=\frac{1}{2}, \mathrm{~B}=0, \mathrm{C}=\frac{-1}{2}$ and $p_{2}=-\frac{1}{3 k_{1}}\left(\frac{p_{1} \mathrm{a}_{-1}}{\mathrm{~b}_{1}}+3\left(p_{3} k_{2}^{2}+p_{4} k_{3}^{2}\right)\right)$.

\section{Conclusion}

Symmetry analysis and modified Exp method were successfully used to obtain new solitary wave solutions for ZK equation. The solutions have physical structures and depend on the real parameters. finally, new type solutions of Riccati were obtained in family 1-4. 


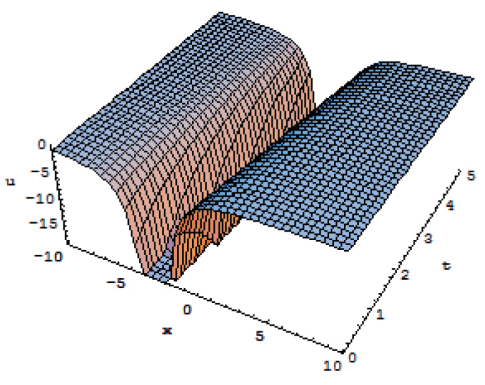

Fig. 1: Singular wave solution of (24), where $\mathrm{p}_{1}=\mathrm{p}_{2}=\mathrm{p}_{3}=\mathrm{p}_{4}=\mathrm{n}=\mathrm{m}_{1}=\mathrm{m}_{2}=\mathrm{m}_{3}=\mathrm{n}_{3}=1, \mathrm{z}=1$.

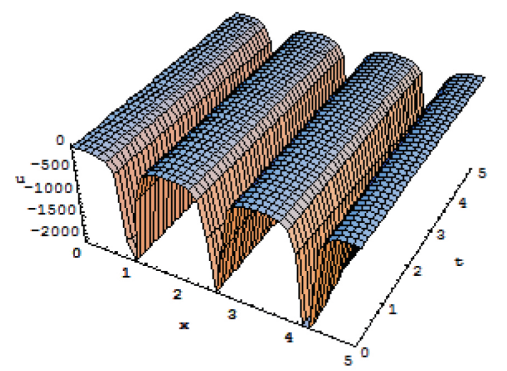

Fig. 3: Periodic solution of (36), where $\mathrm{p}_{1}=\mathrm{p}_{2}=\mathrm{p}_{3}=\mathrm{p}_{4}=\mathrm{m}_{1}=\mathrm{m}_{2}=\mathrm{m}_{3}=\mathrm{n}_{3}=1$, $\mathrm{a}_{0}=\mathrm{k}_{1}=\mathrm{k}_{2}=\mathrm{k}_{3}=1$ and $\mathrm{y}=\mathrm{z}=1$.

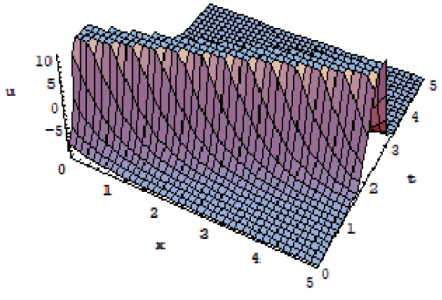

Fig. 2: Periodic solution of (31), where $\mathrm{p}_{1}=\mathrm{p}_{2}=\mathrm{p}_{3}=\mathrm{p}_{4}=\mathrm{m}_{1}=\mathrm{m}_{2}=\mathrm{m}_{3}=\mathrm{n}_{3}=1$, $\mathrm{k}_{1}=\mathrm{k}_{2}=\mathrm{k}_{3}=1$ and $\mathrm{y}=\mathrm{z}=1$.

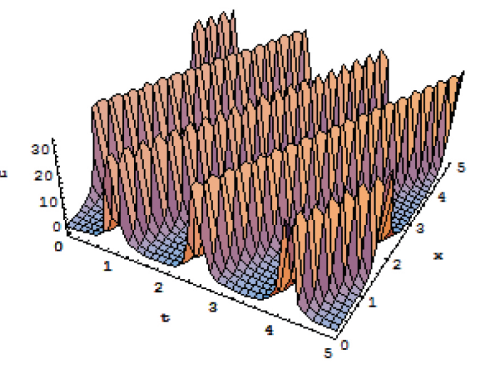

Fig. 4: Periodic solution of (48), where $\mathrm{p}_{1}=\mathrm{p}_{2}=\mathrm{p}_{3}=\mathrm{p}_{4}=\mathrm{m}_{1}=\mathrm{m}_{2}=\mathrm{m}_{3}=\mathrm{n}_{3}=1$, $\mathrm{k}_{1}=\mathrm{k}_{2}=\mathrm{k}_{3}=1$ and $\mathrm{y}=\mathrm{z}=1$.

\section{ACKNOWLEDGMENT}

The authors would like to thank the Deanship of Scientific Research of Majmaah University for supporting this work.

Conflicts of Interest: The author(s) declare that there are no conflicts of interest regarding the publication of this paper.

\section{REFERENCES}

[1] Abdullahi Rashid Adem, Symbolic computation on exact solutions of a coupled Kadomtsev Petviashvili equation: Lie symmetry analysis and extended tanh method, Comput. Math. Appl. 74 (8) (2017), 1897-1902.

[2] Anupma Bansal, Anjan Biswas, Qin Zhou, M.M. Babatin, Lie symmetry analysis for cubic-quartic nonlinear Schrödinger's equation, Optik, 169 (2018), 12-15.

[3] E.M.E.Zayed, K.A.E.Alurrfi, Extended auxiliary equation method and its applications for finding the exact solutions for a class of nonlinear Schrodinger-type equations, Appl. Math. Comput. 289 (2016), 111-131.

[4] M.A.Abdou, A.A.Soliman, On completely integrable coupled Burgers and coupled Korteweg-deVriessystems, Appl. Math Lett. 25 (2012), 2052-2057.

[5] E.M.E.Zayed, Abdul-GhaniAl-Nowehy, Exact solutions and optical soliton solutions for the $(2+1)$-dimensional hyperbolic nonlinear Schrodinger equation, Optik, 127 (12) (2016), 4970-4983.

[6] L.V. Ovsiannikov, Group analysis of differential equations. Moscow: Nauka; 1978.

[7] Olver PJ. Application of lie groups to differential equations. Springer-Verlag; 1986. 
[8] Ibragimov NH, editor. CRC handbook of lie group analysis of differential equations, vols. I, II, III; 1994

[9] G.W. Bluman, S. Kumei, Symmetries and Differential Equations, Springer-Verlag, New York, 1989.

[10] Huaitang Chen, Huicheng Yin, A note on the elliptic equation method, Commun. Nonlinear Sci. Numer. Simul. 13 (2008), $547-553$

[11] V.E. Zakharov, E.A. Kuznetsov, On three-dimensional solitons. Sov. Phys. JETP. 39 (1974), 285-286.

[12] H.A. Biagioni, F. Linares, Well-posedness Results for the Modified Zakharov-Kuznetsov Equation. In: Lupo D., Pagani C.D., Ruf B. (eds) Nonlinear Equations: Methods, Models and Applications. Progress in Nonlinear Differential Equations and Their Applications, Birkhäuser, Basel, 54 (2003), 181-189.

[13] A. A. El-Satar, Similarity Reductions and New Exact Solutions for B-Family Equations, Amer. J. Math. Stat. 2 (3) (2012), 40-43.

[14] M.H.M. Moussa, Similarity Reductions to nonlinear partial differential equation to physical phenomena represented by the Zakharov-Kuznetsovequation, Inter. J. Eng. Sci. 39 (2001), 1565-1575.

[15] T. Ozer, New exact solutions to the CDF equations, Chaos Solitons Fractals, 39 (2007), 1371-1385

[16] M.H.M. Moussa, Similarities, symmetries, singularities and exact solutions for nonlinear system, Ph. D. Thesis, IIT, Delhi, 1991.

[17] A.H. Khater, M.H.M. Moussa, S.F. Abdul-Aziz and H.M.S. Khidr, On the similarity solutions of the variable-coefficient semi-linear hyperbolic equation $\mathrm{u}_{x t}=\alpha(\mathrm{t}) \mathrm{f}(\mathrm{u})$ via a symmetry method, IL Nuovo. Cimento B, 116 (2001), 889-906.

[18] A.H. Khater, W. Malfiet, M.H.M. Moussa, S.F. Abdul-Aziz, On the similarity solutions of the Calogero-Degasperis-Fokas modified KdV equation via symmetry method, J. Phys. Soc. Japan, 72 (10) (2003), 2523-2529.

[19] M.F. El-Sayed, G.M. Moatimid, M.H.M. Moussa, RM El-Shiekh, New exact solutions for the classical Drinfel'd-SokolovWilson equation using the first integral method, Int. J. Adv. Appl. Math. Mech. 1(4) (2014), 52-60.

[20] M. H. M. Moussa, Rehab M. El Shikh, Similarity Reduction and similarity solutions of Zabolotskay-Khoklov equation with a dissipative term via symmetry method, Physica A, 371 (2006), 325-335.

[21] A. A. El-Satar, Symmetry group analysis and similarity solutions for the (2+1)-dimensional coupled Burger's system, Math. Method Appl. Sci. 37 (8) (2014), 1113-1120.

[22] A.A. El-Satar, A study of integrability and symmetry for the $(\mathrm{p}+1)$ th Boltzmann equation via Painlevé analysis and Lie-group method, Math. Method Appl. Sci. 38 (17) (2015), 3670-3677.

[23] Abd El-Halim Ebaid, Generalization of He's Exp-Function Method and New Exact Solutions for Burgers Equation, Z. Naturforsch. A, 64 (2009), 604-608.

[24] Abd El-Halim Ebaid, An improvement on the Exp-function method when balancing the highest order linear and nonlinear terms, J. Math. Anal. Appl. 392 (2012), 1-5.

[25] A. A. Gaber and et., The generalized version of Kudryashov method for nonlinear space-time fractional partial differential equations of Burgers type, Nonlinear Dyn. 95 (2019), 361-368. 